\title{
DISLOCATION OF THE HEAD OF THE RADIUS ASSOCIATED WITH FRACTURE OF THE UPPER END OF THE ULNA IN CHILDREN
}

\author{
S. D. Theodorou, Athens, Greece \\ From the Children's Hospital "A. Kyriakou”, Athens
}

Traumatic dislocation of the head of the radius in children may be either an isolated injury or, more commonly, part of a more complex injury to the elbow, such as the Monteggia fracture-dislocation. In that injury the dislocation of the head of the radius is described as anterior or posterior and the fracture of the ulna affects its shaft (Watson-Jones 1956).

Wise (1941) reported a case of lateral dislocation of the head of the radius associated with incomplete fracture of the olecranon which produced a varus deformity in a seven-year-old boy. He considered it "an unusual Monteggia fracture". Hume (1957) reported three cases of anterior dislocation of the head of the radius associated with undisplaced fracture of the olecranon in children. He differentiated this type of injury from the known Monteggia fracturedislocation. Beddow and Corkery (1960) described two cases, and Wright (1963) added five new cases.

The purpose of this paper is to describe five new cases of this injury which has received such little attention in the literature. The first three cases are more interesting because the injury to the elbow was associated with fracture of the lower end of the radius and ulna. We have not found this particular type of injury in the literature.

\section{CASE REPORTS}

Case 1-A boy aged twelve years fell off a horse on to the left arm and was admitted to the hospital two days later. Radiographs (Fig. 1) showed an undisplaced fracture of the upper end of the ulna with some varus deformity, and lateral dislocation of the head of the radius. There was also a fracture of the lower part of the radius and ulna with slight postero-lateral displacement. Under general anaesthesia the dislocated head of the radius was easily reduced but slight varus deformity of the upper end of the ulna was accepted. The deformity of the forearm was satisfactorily corrected. A plaster was applied and was kept on for four weeks. After the removal of the plaster the boy was instructed in exercises. Two months later he had full range of movements and no deformity in the elbow although radiographs showed slight varus deformity of the upper part of the ulna (Fig. 2).

Case 2-A ten-year-old boy fell off a tree on to his left arm and was admitted to the hospital on the same day. Radiographs showed a fracture of the olecranon with slight displacement, lateral dislocation of the head of the radius and subperiosteal fracture of the lower part of the radius and ulna with slight postero-lateral deformity (Fig. 3). The dislocation was reduced and the deformities were corrected on the same day under general anaesthesia and an above-elbow plaster was applied and kept on for four weeks. Radiographs ten weeks after the injury showed full reduction of the radial dislocation and no residual deformity (Fig. 4). The range of movement of the elbow and forearm was full.

Case 3-A boy aged twelve years fell off a tree about 3 metres high on to his left arm and was admitted to the local hospital where an attempt at reduction was made without success. He was referred to us three days later. Radiographs showed fracture of the upper end of the ulna, lateral dislocation of the radial head and fracture of the lower part of the radius and ulna on the same side with displacement (Fig. 5). The fracture of the forearm was not discovered until the boy was taken to the theatre for reduction. The dislocation of the radial head was reduced without difficulty and the deformities of the olecranon and the lower part of the forearm were corrected. Some displacement of the olecranon fracture was accepted. A check radiograph a week later showed that the position was maintained. This boy has not attended for follow-up, but the plaster was removed by the local doctor after four weeks. A radiograph eight months later shows good reduction (Fig. 6). He has full range of movements in the elbow and forearm. 


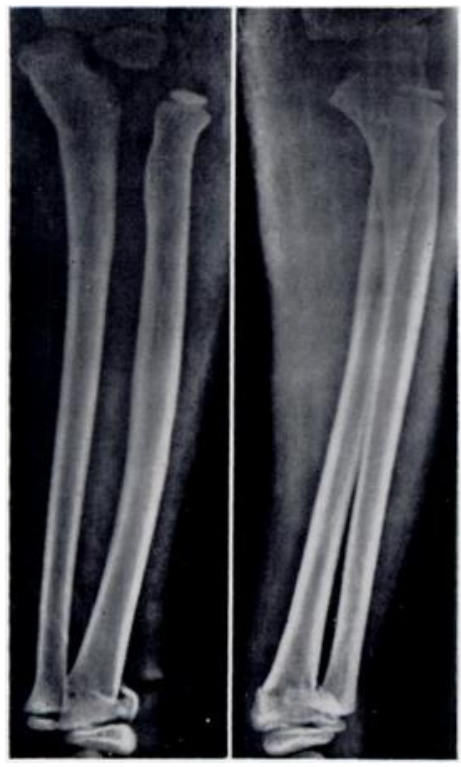

Fig. 1

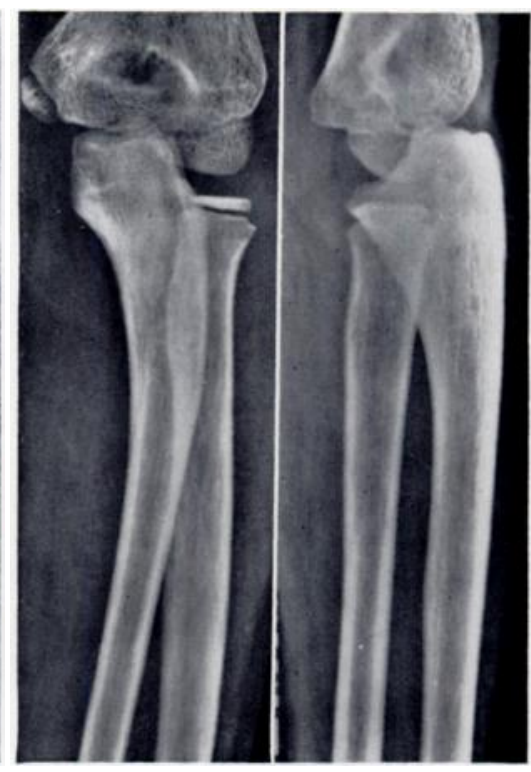

FIG. 2

Case 1. Figure 1-Before reduction. Incomplete fracture of the upper end of the ulna associated with lateral dislocation of the head of the radius. Fracture of the lower part of the radius on the same side. Figure 2-Two months after closed reduction there is slight varus deformity of the upper end of the ulna but the radial head is fully reduced.

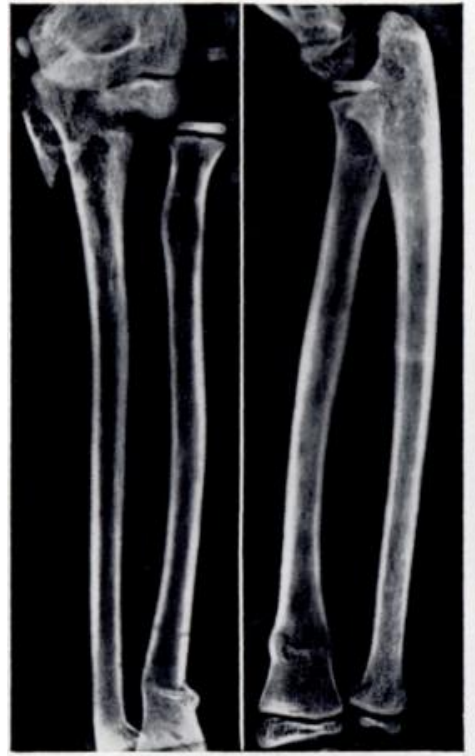

FiG. 3

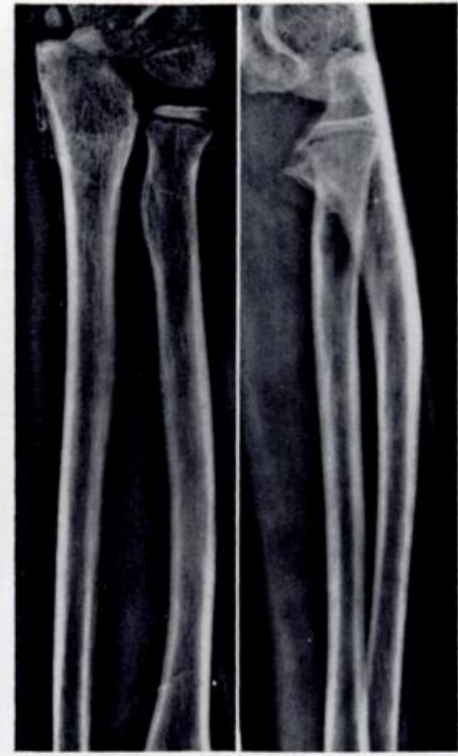

Fig. 4

Case 2. Figure 3-Before reduction. Fracture of the olecranon with some displacement and lateral dislocation of the head of the radius. Incomplete fracture of the lower part of the radius and ulna with some deformity on the same side. Figure 4-Two and a half months after closed reduction. No residual deformity. 


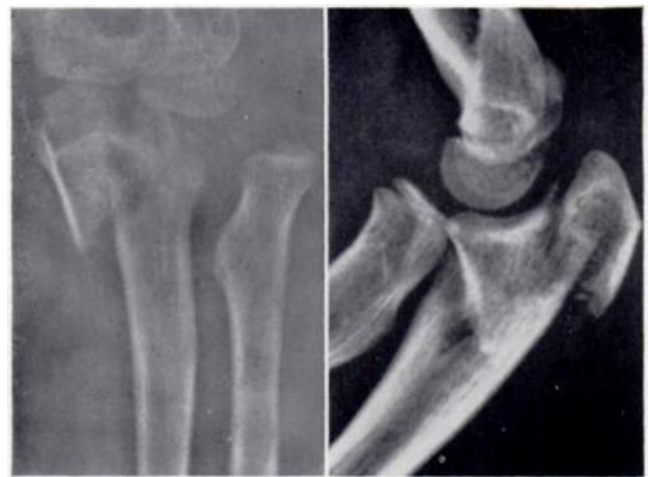

FiG. 5

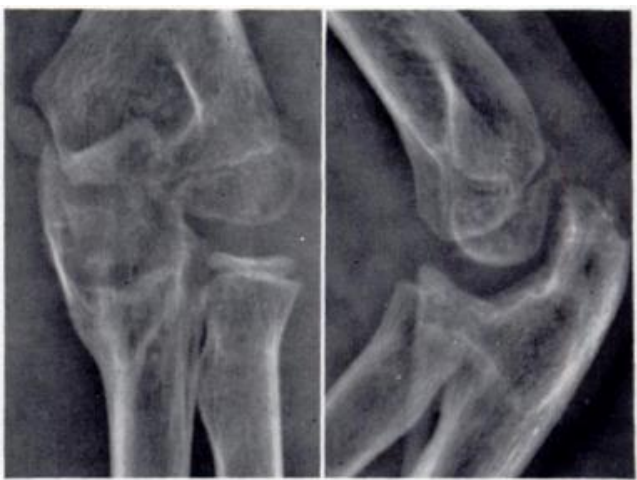

Fig. 6

Case 3. Figure 5-Before reduction. Fracture of the upper end of the ulna and lateral dislocation of the head of the radius. Figure 6-Eight months after closed reduction.

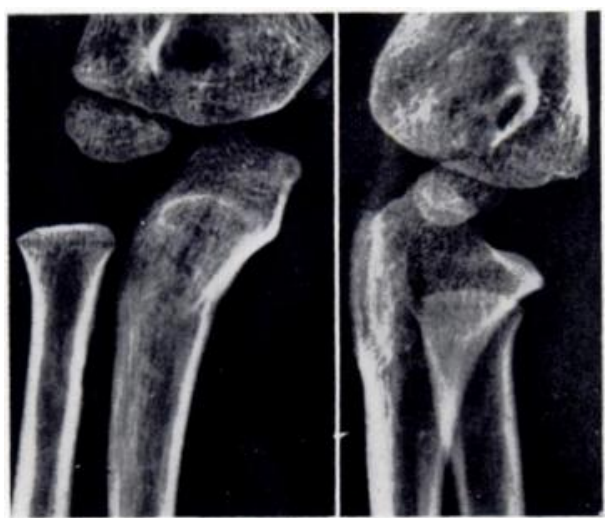

FIG. 7

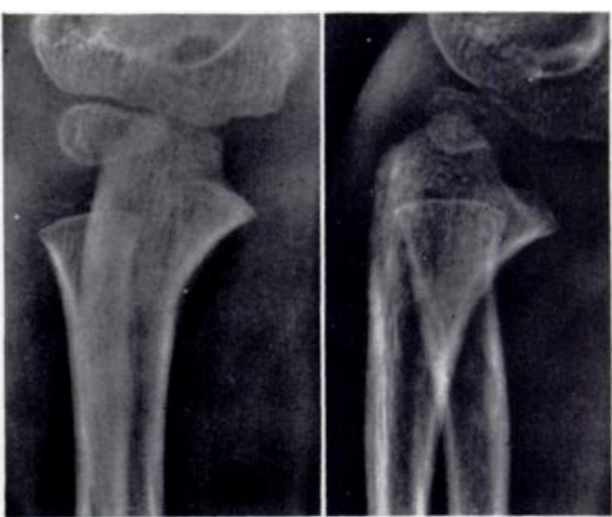

FiG. 8

Case 4. Figure 7-Before reduction. Greenstick fracture of the upper end of the ulna with varus deformity, and lateral dislocation of the head of the radius. Figure 8-Six weeks after closed reduction. No residual deformity.

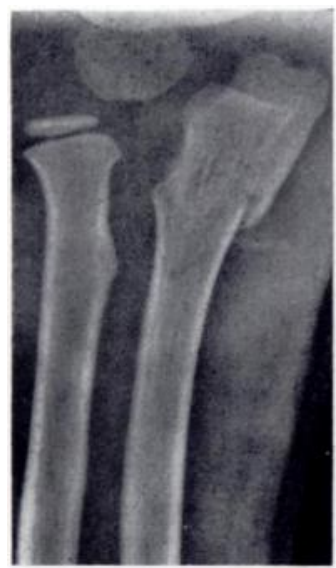

FiG. 9

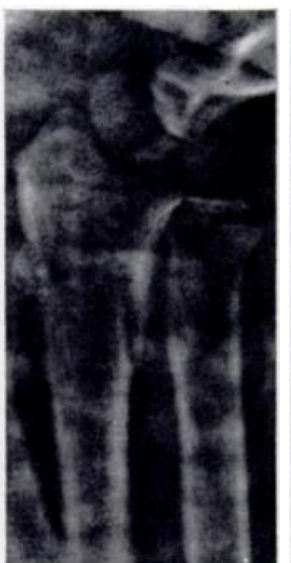

Fig. 10

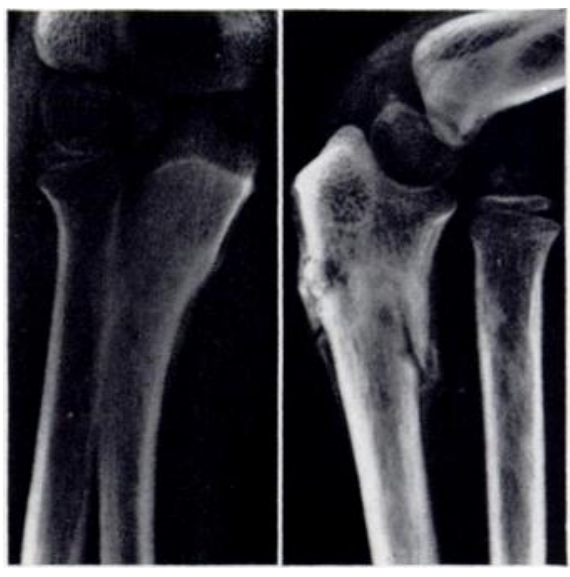

Fig. 11

Case 5. Figure 9-Before reduction. Fracture of the upper end of the ulna with varus deformity and lateral dislocation of the head of the radius. Figure 10-In plaster after closed reduction.

Position good. Figure 11-After four weeks. Anterior dislocation of the head of the radius. Note calcification over radial head. 
Case 4-A boy aged six years fell off a tree and injured his right arm. He was admitted to hospital two days later. Radiographs showed an undisplaced fracture of the olecranon with varus deformity and lateral dislocation of the head of the radius (Fig. 7). Under general anaesthesia the dislocation of the head of the radius was reduced and the deformity of the olecranon improved. A plaster was applied and retained for four weeks. Five weeks after the injury the boy had a full range of movements and radiographs of the elbow showed almost no abnormality (Fig. 8).

Case 5-A six-year-old boy was kneeling on the floor when his elder brother jumped suddenly on his back. He was admitted to hospital for an injury to his right elbow. Radiographs showed a fracture of the upper end of the ulna with slight displacement and lateral dislocation of the head of the radius (Fig. 9). The dislocation of the head of the radius was reduced and the deformity of the olecranon was corrected the next day. Radiographs showed good reduction (Fig. 10). A plaster-of-Paris splint was applied and retained for four weeks. After the removal of plaster radiographs showed that slight antero-lateral dislocation of the radius persisted (Fig. 11). There was also a small calcification over the radial head. The range of movements was good. Open reduction was not suggested. Five months later the picture was unchanged. There was slight limitation of rotation of the forearm.

\section{DISCUSSION}

In the eleven cases of this injury so far reported there are differences regarding the exact type of dislocation of the head of the radius and the fracture of the upper end of the ulna (Fig. 12). Hume (1957) described the dislocation of the radial head as anterior and the fracture of the olecranon as undisplaced. In fact, the dislocation is anterior in his second and third cases. His first case is different: the dislocation of the radial head is lateral and the fracture of the upper end of the ulna shows varus deformity. The case reported by Wise (1941), the two cases of Beddow and Corkery (1960), Wright's (1963) Case 3 and our Cases 1 and 4 are similar. In the remaining three of our cases, although there is definite varus deformity in the elbow and lateral or antero-lateral dislocation of the head of the radius, the fracture of the upper end of the ulna is more severe, and there is some displacement. In Hume's (1957) Cases 2 and 3 there is a small avulsion fracture of the olecranon without varus deformity of the upper end of the ulna, and anterior dislocation of the head of the radius. The dislocation of the radial head in Wright's Cases 1 and 2 is also anterior but the fracture of the upper end of the ulna is almost complete and there is some anterior displacement of the distal fragment. It is obvious that all these cases represent hyperextension injuries to the elbow with anterior dislocation of the head of the radius. Wright's Cases 4 and 5 represent more complex injuries to the elbow and should not, in our view, be included in the same category.

Mechanism of injury-Our first three cases give some information regarding the mechanism of the injury. It is certain that all these children fell on to the outstretched hand. The force was transmitted to the extended elbow. The exact type of dislocation of the head of the radius (anterior, lateral or antero-lateral) and the deformity of the upper end of the ulna depends upon the angulation of the elbow at the time of the injury. What is the position of the bones of the forearm at the time of the injury? The mechanism described by Evans (1949) for the Monteggia fracture, a fall forwards on to the outstretched hand with the elbow semiflexed and the forearm in forced pronation by the rotation on it of the body, does not correspond to the features of the injury under discussion.

Because children are on the whole unreliable witnesses, we were not able to get a definite description of the position of the arm during the injury. In any case, all of them, except the boy in Case 5, fell from a height on to the outstretched hand. From the analysis of the eleven cases of the literature, it is obvious that this is the most usual type of injury producing this lesion (Fig. 12). It is therefore probable that a fall sideways with the forearm in supination or in slight pronation, and the hand in dorsiflexion, might explain the production of this lesion. In none of our cases did any local signs suggest a possible direct blow to the elbow.

Sex incidence-All our children were boys. The predominance of boys is also clear in the eleven reported cases in the literature (Fig. 12).

VOL. 51 B, NO. 4, NOVEMBER 1969 
S. D. THEODOROU

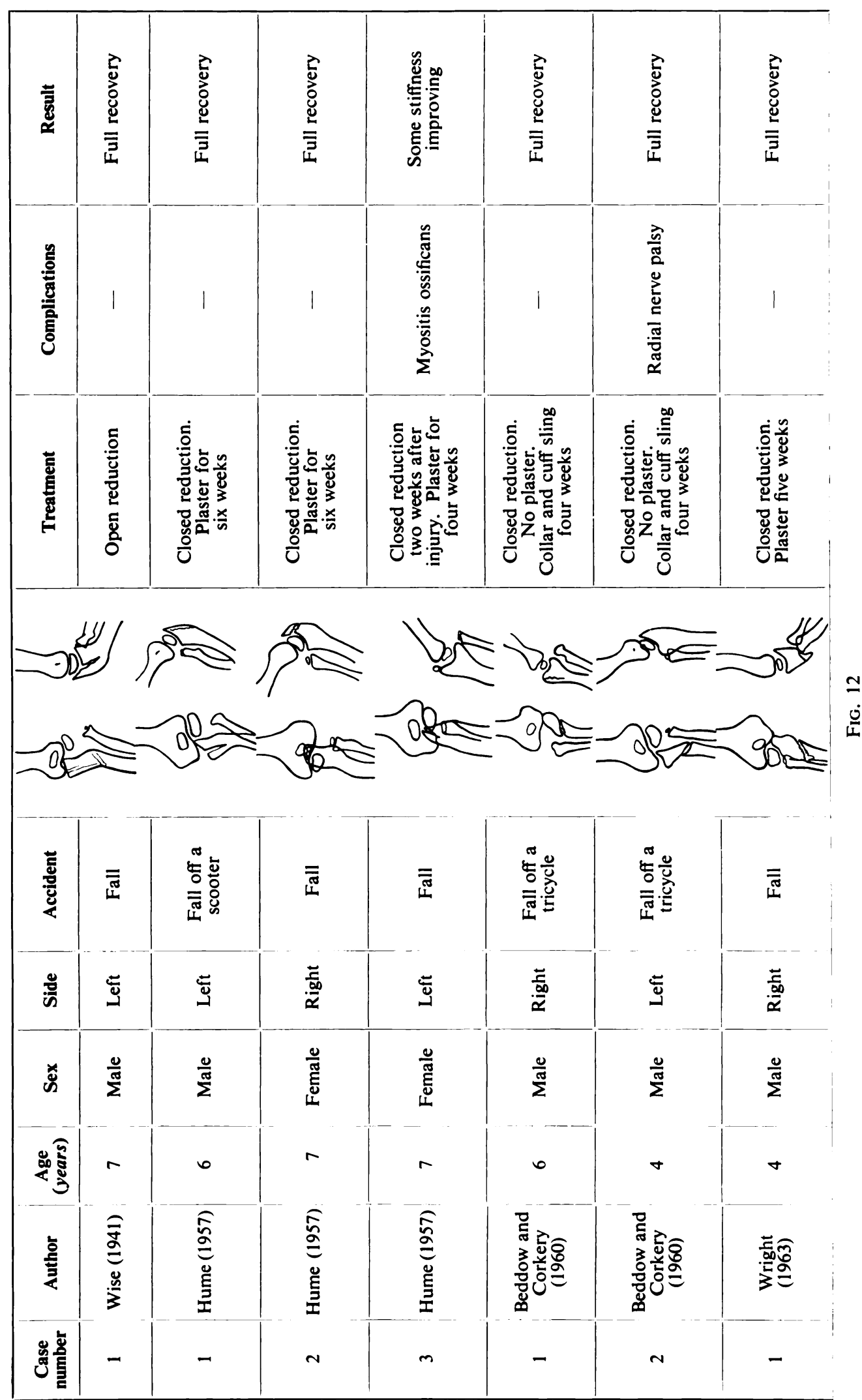

THE JOURNAL OF BONE AND JOINT SURGERY 
DISLOCATION OF HEAD OF RADIUS WITH FRACTURE OF UPPER END OF ULNA IN CHILDREN 705

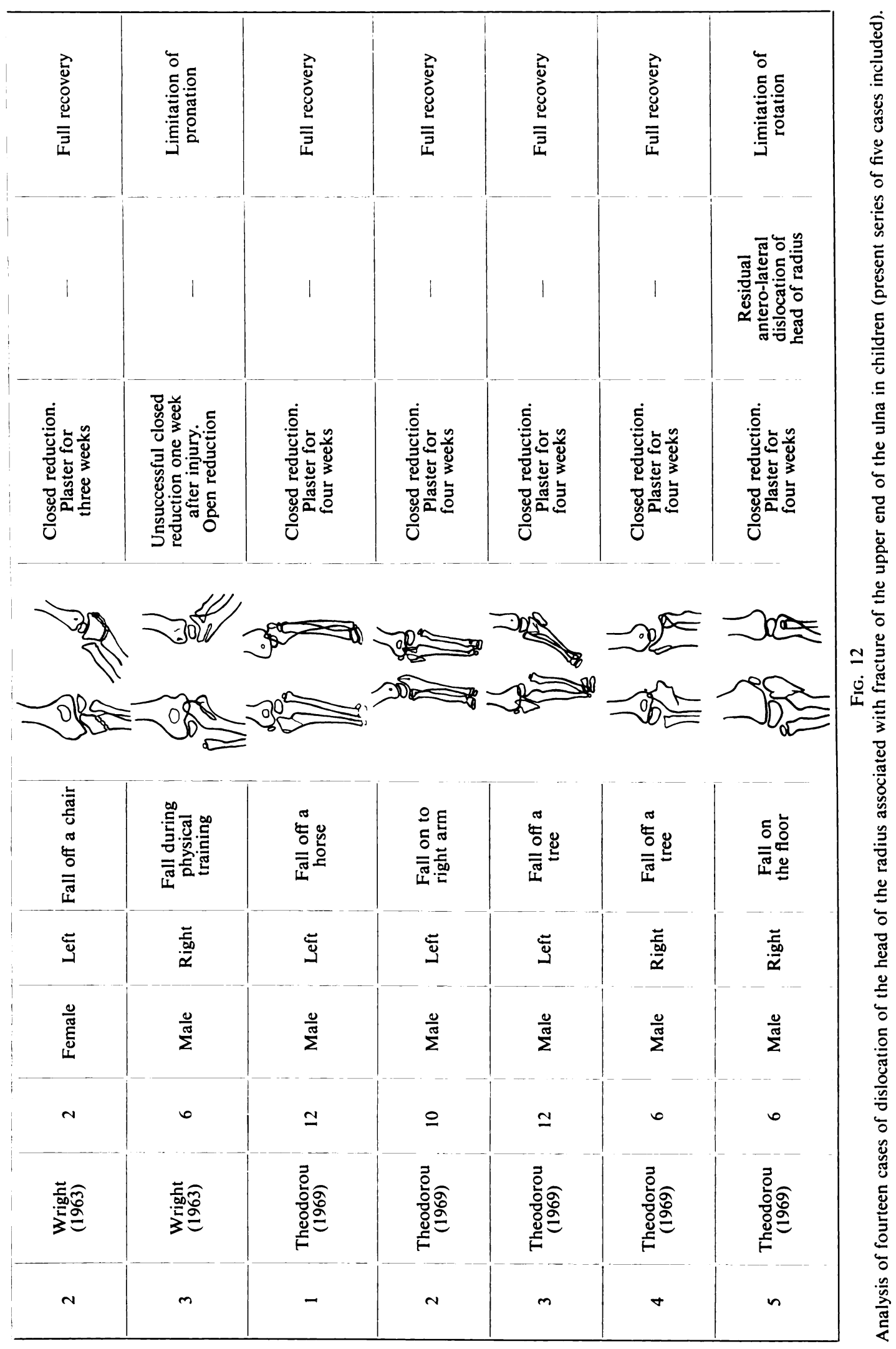

VOL. 51 B, NO. 4, NOVEMBER 1969 
Treatment-Closed reduction seems to be the ideal method of treatment. The lateral deformity of the upper end of the ulna is corrected and the dislocation of the head of the radius reduced by pressure and pronation of the forearm. Great attention must be given to the complete reduction of the head of the radius by checking the position of the head under radiographic control. If reduction is impossible, or in neglected cases, open reduction may be necessary.

Complications-Wise's case was treated by open reduction. It is not mentioned whether or not this followed an unsuccessful attempt at closed reduction. In Hume's Case 3 the dislocation of the head of the radius was only discovered two weeks after the injury and therefore open reduction was necessary. Open reduction was also performed in Wright's Case 3 a week after an unsuccessful closed reduction. Some limitation of pronation and supination of the forearm was observed. Beddow and Corkery's (1960) Case 2 presented a temporary radial palsy. In our fifth case, although the reduction of the head of the radius looked good (Fig. 10), radiographs after the removal of plaster four weeks later showed slight antero-lateral dislocation of the radial head and some calcification over it. Open reduction was not performed.

\section{SUMMARY AND CONCLUSIONS}

1. Five cases of dislocation of the head of the radius associated with fracture of the upper end of the ulna in children are reported. It is important that fractures of the upper end of the ulna, especially those with some varus deformity in children, should arouse suspicion of the possible co-existence of a dislocation of the head of the radius.

2. The association of the injury to the elbow with a fracture of the lower end of the radius and ulna in three of our cases indicates the necessity for keeping in mind the possibility of this combination.

3. Closed reduction seems to be the best method of treatment.

4. Our five cases were seen during a period of only three years. We therefore believe that this injury is not as unusual as appears from the literature.

\section{REFERENCES}

Beddow, F. H., and Corkery, P. H. (1960): Lateral Dislocation of the Radio-humeral Joint with Greenstick Fracture of the Upper End of the Ulna. Journal of Bone and Joint Surgery, 42-B, 782.

Evans, E. M. (1949): Pronation Injuries of the Forearm. Journal of Bone and Joint Surgery, 31-B, 578.

Hume, A. C. (1957): Anterior Dislocation of the Head of the Radius Associated with Undisplaced Fracture of the Olecranon in Children. Journal of Bone and Joint Surgery, 39-B, 508.

Watson-Jones, Sir R. (1956): Fractures and Joint Injuries. Fourth edition, Volume II, p. 572. Edinburgh and London: E. \& S. Livingstone Ltd.

WISE, R. A. (1941): Lateral Dislocation of the Head of the Radius with Fracture of the Ulna. Journal of Bone and Joint Surgery, 23, 379.

WRIGHT, P. R. (1963): Greenstick Fracture of the Upper End of the Ulna with Dislocation of the Radiohumeral Joint or Displacement of the Superior Radial Epiphysis. Journal of Bone and Joint Surgery, 45-B, 727. 